\title{
Higher Emotion-Related Distress in Patients with Fibromyalgia versus Chronic Neuropathic Pain and Healthy Controls: Is It a Primary Affective Disorder?
}

\author{
Ksenija Vucurovic 1,2,3*, Delphine Raucher-Chéné1,4, Clémentine Dupont-Gaudin", \\ Philippe Peruzzi ${ }^{2}$, Jean-François Visseaux ${ }^{3}$, Arthur Kaladjian1,4, \\ Christine-Vanessa Cuervo-Lombard 5
}

${ }^{1}$ Laboratoire Cognition, Santé, Société (C2S), Reims Champagne-Ardenne University, Reims, France

${ }^{2}$ Neurosurgery Department, Pôle Tête et Cou, Centre Hospitalo-Universitaire de Reims, Reims, France

${ }^{3}$ Centre Rémois de Psychothérapie et Neuromodulation (CRPN), Reims, France

${ }^{4}$ Pôle Universitaire de Psychiatrie, CHU de Reims, EPSM Marne, Reims, France

${ }^{5}$ Center for Studies and Research on Health Psychology and Psychopathology (CERPPS), Department of Psychology, Toulouse 2 Jean Jaurès University, Toulouse, France

Email: *kvucurovic@chu-reims.fr

How to cite this paper: Vucurovic, K., Raucher-Chéné, D., Dupont-Gaudin, C., Peruzzi, P., Visseaux, J.-F., Kaladjian, A., \& Cuervo-Lombard, C.-V. (2020). Higher Emotion-Related Distress in Patients with Fibromyalgia versus Chronic Neuropathic Pain and Healthy Controls: Is It a Primary Affective Disorder? Psychology, 11, 1547-1558. https://doi.org/10.4236/psych.2020.1110098

Received: September 28, 2020

Accepted: October 25, 2020

Published: October 28, 2020

Copyright $\odot 2020$ by author(s) and Scientific Research Publishing Inc. This work is licensed under the Creative Commons Attribution International License (CC BY 4.0).

http://creativecommons.org/licenses/by/4.0/

\section{(c) (i) Open Access}

\begin{abstract}
Fibromyalgia is central sensitization pain disorder with various psychological symptoms. Our aim is to compare trait paranoia, self-esteem and impulsivity between fibromyalgia patients (FM), chronic neuropathic pain sufferers (CNP) and healthy control. We administrated the Mini International Neuropsychologic Interview to $30 \mathrm{FM}, 27 \mathrm{CNP}$ and $22 \mathrm{HC}$. All participants completed the Paranoia, Rosenberg Self-Esteem and Short UPPS-P Impulsivity Behavior Scales, Beck Depression and State-Trait Anxiety inventories. Patients provided pain ratings and completed the French version of the McGill Pain Questionnaire and Pain Catastrophizing Scale. An analysis of variance compared the three groups, with adjustment for psychological distress, pain severity and education level. Anxiety-related psychiatric comorbidities were more prevalent in FM. Depression and state anxiety were higher in both CNP and FM, while trait anxiety was higher in FM compared to two other groups. Paranoia scores were significantly higher among FM than among HC, with intermediate scores in CNP. These group differences remained after adjustment for psychological distress, pain severity and education level. Lower self-esteem and higher negative urgency in patients with FM disappeared when results were adjusted for psychological distress. FM described sensory and affective aspects of pain as more severe and displayed higher pain catastrophizing than CNP group. These results suggest that emotion-related dis-
\end{abstract}


tress is higher in FM than in CNP.

\section{Keywords}

Paranoia, Chronic Pain, Self-Esteem, Impulsivity

\section{Introduction}

Fibromyalgia (FM), described as a prototypal central chronic pain disorder, is characterized by widespread chronic pain as its core symptom, sleep disturbances, psychological distress, fatigue, cognitive dysfunction, and somatic symptoms (Häuser et al., 2015). It occurs in $2 \%$ - $4 \%$ of general population, mostly in women, and can induce severe functional disability (Häuser et al., 2015). The underlying pathophysiology is still poorly understood, even though an increasing number of researchers are interested in this challenging concept of complex imbrication between physical symptoms and psychological distress. The hypotheses of existence of FM-prone personality or fibromyalgia as a form of masked depression appear as still unresolved topic (Häuser \& Fitzcharles, 2018). It has been suggested that an overall deficit in sustaining positive emotions in face of pain in FM is one of perpetuating factors of the illness (Rosselló et al., 2015), but other emotion-related processes have as yet been poorly explored in this population.

Emotion-related processes would refer to the psychological, psychopathological and neurological mechanisms by which distressed emotional reactions in individuals are converted to non-distressed reactions (Baker, 2007). Emotion-related processes strongly influence emotional stated such as anxiety and depression, but also mental, behavioral and physical health of the individual (Lumley et al., 2011). There are consistent literature data in cognitive sciences that highlighted the importance of affective state and emotions in learning (Baker et al., 2010; Prokofieva et al., 2019; Woolf et al., 2009). Yet, learning and memory are strongly involved in psychopathology of chronic pain and some authors suggest that "chronic pain is pain that does not extinguish its memory trace" (Mansour et al., 2014). Therefore, better understanding of emotion-related processes associated with chronic pain is fundamental for improving clinical interventions. Moreover, direct comparison of these processes in two distinct patients' groups, chronic neuropathic pain sufferers (CNP) and FM, could give us insight into psychopathological mechanisms of vulnerability to central sensitization for pain.

Here we adopted clinical-based perspective to approach relevant emotion-related processes in chronic pain disorders. Our clinical experience guided our choice of clinical measures to be done, and rationale of our choice was inspired by DSM-5 pathological personality traits. We decided to measure paranoia as facet of antagonism, self-esteem of negative affectivity and impulsivity of disinhibition (Hopwood et al., 2013). Paranoia is defined as unjustified distrust and suspicion of others (Freeman et al., 2012). Trait paranoia has been associated with emotional deregulation, social isolation, fewer activities, and a feeling of powerlessness in 
nonclinical individuals (Freeman et al., 2012). It is highly prevalent in patients with mood disorders, particularly depression accompanied by somatic complaints and sensory disturbances (Freeman et al., 2012), and as such appears as pertinent clinical dimension to be measured in chronic pain patients. In addition, patients with chronic pain have been found to have higher trait paranoia than healthy individuals (Conrad et al., 2007) but no direct distinction has so far been made between FM and other chronic pain conditions on this personality dimension.

It has been postulated that trait paranoia is driven by low self-esteem and negative emotions (Thewissen et al., 2011). Self-esteem refers to thoughts, feelings, and evaluations about the self. Low self-esteem was previously reported in FM (Galvez-Sánchez et al., 2018). Low explicit self-esteem may be a vulnerability trait for a wide range of psychiatric conditions, from personality to affective disorders and psychosis (Bentall et al., 2009). It was suggested that positive self-esteem contributes to individual's intrinsic motivation that further facilitates success in cognitive tasks (Galvez-Sánchez et al., 2018). Lack of self-esteem may thus constitute another possible affective feature involved in failure of FM patients to provide sustained effort in cognitive tasks (Bar-On Kalfon et al., 2016). Given this observation, it remains important to compare groups of chronic pain patients on this clinical dimension. The third dimension that we chose to measure is impulsivity. It is defined as deficient control over behavior, and manifests itself as a preference for immediate but smaller rewards over larger delayed ones. High impulsivity therefore reduces motivation to engage in tasks that require sustained effort (Zisner \& Beauchaine, 2016) and further generates negative emotions. Higher impulsivity associated to higher prevalence of attention-deficit hyperactivity disorder was previously reported in FM (Yilmaz \& Tamam, 2018). Importantly, Vest et al., 2016 suggested that chronic pain sufferers who react impulsively to negative mood states and cravings may be prone to opioid misuse in clinical practice.

Variability in nature of the symptoms reported by FM patients makes difficult our attempts to understand psychological mechanisms behind the illness. Dimensional approaches could therefore be useful to ascertain the precise psychopathological features of FM, particularly for emotion-related processes.

Our first aim was to assess trait paranoia, impulsivity and self-esteem in patients with FM, comparing them with CNP and healthy controls (HC) in order to elucidate if FM patients differed from others on these personality dimensions highly predictive of poor emotional coping. Further, we intended to analyze correlation between these dimensions, pain severity and psychological distress in patients' groups in order to explore how personality dimensions of emotion-related processes associate with pain experience in patients.

Based on literature data and our clinical experience, we expected to observe higher paranoia and impulsivity and lower self-esteem in the FM group than in either the CNP or HC groups. In addition, we expected that higher scores of paranoia and impulsivity and lower self-esteem would correlate with higher pain intensity, in particular on the affective aspects of the pain evaluation. 


\section{Material and Method}

\subsection{Participants}

We recruited 30 patients diagnosed with FM, according to American College of Rheumatology 2010 criteria, 27 patients with CNP eligible for invasive chronic motor cortex stimulation in a neurosurgical department and $22 \mathrm{HC}$. All patients reported experiencing pain for more than 6 months. Non-inclusion criteria for all groups were being under 18 years of age, any other chronic pain condition, current or past psychotic disorder, neurological disorder, and autoimmune disease or any other severe medical condition that might affect the clinical evaluation. We also excluded participants from the HC group if they took medication for pain or reported any chronic pain.

All participants were native French speakers. The research was conducted in accordance with the Declaration of Helsinki, and was approved by the local ethics committee. All participants gave their written informed consent before being included in the study.

\subsection{Clinical Measures}

We administered the Mini International Neuropsychiatric Interview (MINI) version 5.0 (Sheehan et al., 1998), a semi-structured clinical interview based on the DSM-IV Axis I diagnostic criteria, to assess mental disorder comorbidity.

All participants completed validated French versions of the Beck Depression Inventory-II (BDI-II; Beck et al., 1996) to collect a subjective measure of depression, the State Trait Anxiety Inventory (STAI; Spielberger et al., 1984) to assess current anxiety and propensity to anxiety, the Paranoia Scale (PS; Fenigstein \& Vanable, 1992) to obtain a dimensional measure of paranoia (Cronbach's alphas: FM: 0.73; HC: 0.90; CNP: 0.81), the Rosenberg Self-Esteem Scale (RSES; Rosenberg, 1965) gauging overall explicit self-esteem (Cronbach's alpha: FM: 0.82; HC: 0.85; CNP: 0.76), and the short version of the UPPS-P Impulsive Behavior Scale that evaluates negative urgency, positive urgency, lack of premeditation, lack of perseverance, and sensation seeking (Billieux et al., 2012; FM: $\alpha=0.65$; HC: $\alpha=$ 0.77; CNP: $\alpha=0.78$ ).

Patients with FM and CNP also provided ratings on a visual numerical scale (VNS) ranging from 0 (No pain) to 10 (Worst pain imaginable) for intensity of current pain and mean and maximum pain during the week before assessment. Pain was also measured with the French version of the McGill Pain Questionnaire (MPQ; Melzack, 1975), the Questionnaire de Saint Antoine composed of 58 pain descriptor items divided into 16 categories ( 9 for sensory dimension and 7 for affective dimension), where patients have to choose the best descriptor of their pain and rate its intensity, and the validated French version of Pain Catastrophizing Scale (PCS; Sullivan et al., 1995), where patients indicate the degree to which they endorse 13 items (thoughts or feelings when experiencing pain) assessing rumination, magnification, and helplessness. 


\subsection{Statistical Analysis}

All the statistical analyses were conducted using SPSS Statistics Windows version 17.0. We ran an analysis of variance (ANOVA) to compare the three groups on clinical assessment scores and sociodemographic characteristics. Whenever a significant main effect of group was found, we conducted post hoc analyses with the Bonferroni test $(p<0.05)$ to evaluate between-group differences. Pairwise comparisons were carried out with the t test or chi-square test. Pearson's correlation coefficients were used to investigate the relations between PS, RSES and UPPS-P scores, pain parameters (pain duration, pain intensity, MPQ and PCS scores and sub scores), and psychological distress (BDI-II, STAI) in a whole participants sample. An analysis of covariance (ANCOVA) was used to evaluate the influence of psychological distress (BDI, STAI) and pain severity (duration, intensity) as covariates on each dependent variable (PS, RSES, UPPS-P scores) for any statistically significant Pearson's correlations.

\section{Results}

\subsection{Demographic and Clinical Data}

Data and the results of statistical analyses of the sociodemographic variables and psychological distress, pain severity, trait dimensions and MINI assessment scores are detailed in Table 1.

Table 1. Between-group comparisons on sociodemographic and clinical variables.

\begin{tabular}{|c|c|c|c|c|c|c|}
\hline & $\mathrm{HC}(n=22)$ & $\mathrm{CNP}(n=27)$ & $\mathrm{FM}(n=30)$ & \multicolumn{3}{|c|}{ Analyses } \\
\hline & Mean $(S D)$ & Mean $(S D)$ & Mean $(S D)$ & $\mathrm{F}(A N O V A)$ & $p$ & Post hoc \\
\hline Age in years & $50.73(7.8)$ & $47.67(9.9)$ & $49.83(8.3)$ & 0.817 & 0.445 & - \\
\hline Education in years & $11.59(1.7)$ & $10.30(1.5)$ & $11.66(1.4)$ & 6.695 & 0.002 & $\mathrm{CNP}<\mathrm{FM}, \mathrm{HC}$ \\
\hline BDI-II & $5.45(5.4)$ & $13.52(10.1)$ & $30.3(10.0)$ & 52.827 & $<0.0001$ & $\mathrm{HC}<\mathrm{CNP}<\mathrm{FM}$ \\
\hline STAI-State & $28.14(8.8)$ & $39.41(14.1)$ & $41.80(17.1)$ & 6.405 & 0.002 & $\mathrm{HC}<\mathrm{CNP}, \mathrm{FM}$ \\
\hline STAI-Trait & $32.45(8.5)$ & $36.78(14.1)$ & $57.77(10.9)$ & 51.476 & $<0.0001$ & $\mathrm{HC}, \mathrm{CNP}<\mathrm{FM}$ \\
\hline Paranoia Scale & $29.55(11.7)$ & $40.59(13.7)$ & $59.60(11.5)$ & 39.954 & $<0.0001$ & $\mathrm{HC}<\mathrm{CNP}<\mathrm{FM}$ \\
\hline RSES & $36(5.1)$ & $34.41(5.2)$ & $24.13(6.5)$ & 34.959 & $<0.0001$ & $\mathrm{FM}<\mathrm{CNP}, \mathrm{HC}$ \\
\hline UPPS-P negative urgency & $8.09(3.0)$ & $9.59(4.1)$ & $10.60(3.3)$ & 3.441 & 0.037 & $\mathrm{HC}, \mathrm{CNP}<\mathrm{FM}$ \\
\hline UPPS-P positive urgency & $8.91(2.6)$ & $9.89(3.4)$ & $10.56(2.6)$ & 2.059 & 0.135 & - \\
\hline UPPS-P lack of premeditation & $6.73(2.1)$ & $6.48(2.4)$ & $7.76(2.9)$ & 2.047 & 0.136 & - \\
\hline UPPS-P lack of perseverance & $6.36(2.2)$ & $5.93(2.4)$ & $7.63(3.4)$ & 2.677 & 0.075 & - \\
\hline \multirow[t]{2}{*}{ UPPS-P sensation seeking } & $9.04(3.7)$ & $7.89(3.3)$ & $7.80(2.6)$ & 1.131 & 0.328 & - \\
\hline & & & & t-test & $p$ & \\
\hline VNS current pain & - & $6.37(2.1)$ & $5.60(2.0)$ & 1.403 & 0.166 & - \\
\hline VNS mean pain & - & $7.56(1.6)$ & $7.46(1.4)$ & 0.221 & 0.826 & - \\
\hline VNS maximum pain & - & $8.56(1.3)$ & $8.60(1.2)$ & -0.131 & 0.896 & - \\
\hline MPQ sensory pain & - & $24.33(5.9)$ & $28.16(5.6)$ & -2.511 & 0.015 & - \\
\hline MPQ affective pain & - & $17.41(5.9)$ & $22.40(4.8)$ & -3.505 & 0.001 & - \\
\hline PCS rumination & - & $10.74(4.8)$ & $12.10(3.1)$ & -1.271 & 0.209 & - \\
\hline
\end{tabular}




\section{Continued}

\begin{tabular}{|c|c|c|c|c|c|c|}
\hline PCS magnification & - & $4.44(3.5)$ & $6.76(2.8)$ & -2.773 & 0.007 & - \\
\hline PCS helplessness & - & $13.33(7.1)$ & $17.73(4.8)$ & -2.773 & 0.007 & - \\
\hline Pain duration (years) & - & $7.26(11.5)$ & $14.00(11.5)$ & 2.704 & 0.009 & - \\
\hline MQS III & - & $11.21(11.3)$ & $18.52(12.8)$ & 1.284 & 0.027 & - \\
\hline MINI & $\%(n)$ & $\%(n)$ & $\%(n)$ & $\mathrm{F}(A N O V A)$ & $p$ & Post hoc \\
\hline Current depressive disorder & $4.55(1)$ & $7.41(2)$ & $36.67(11)$ & 46.531 & $<0.0001$ & $\mathrm{HC}<\mathrm{CNP}<\mathrm{FM}$ \\
\hline \multirow[t]{2}{*}{ Past depressive disorder } & $18.18(4)$ & $44.44(12)$ & $33.33(10)$ & 12.419 & 0.002 & $\mathrm{HC}<\mathrm{CNP}, \mathrm{FM}$ \\
\hline & & & & $X^{2}$ & $p$ & \\
\hline Bipolar disorder & - & $0(0)$ & $3.33(1)$ & 3.387 & 0.066 & - \\
\hline Current panic disorder & - & $0(0)$ & $10(3)$ & 10.526 & 0.001 & - \\
\hline Agoraphobia & - & $3.70(1)$ & $16.67(5)$ & 9.195 & 0.002 & - \\
\hline Current social phobia & - & $11.11(3)$ & $10(3)$ & 0.236 & 0.627 & - \\
\hline Current OCD & - & $0(0)$ & $3.33(1)$ & 3.387 & 0.066 & - \\
\hline Current PTSD & - & $0(0)$ & $0(0)$ & - & - & - \\
\hline Current GAD & - & $11.11(3)$ & $56.67(17)$ & 46.323 & $<0.0001$ & - \\
\hline Anorexia nervosa & - & $0(0)$ & $3.33(1)$ & 3.387 & 0.066 & - \\
\hline Bulimia nervosa & - & $0(0)$ & $0.10(3)$ & 0.526 & 0.001 & - \\
\hline
\end{tabular}

$\mathrm{HC}=$ Healthy Controls; CNP = Patients with Chronic Neuropathic Pain; FM = Patients with Fibromyalgia; BDI-II = Beck Depression Inventory-II; STAI = Spielberger State Trait Anxiety Inventory; RSES = Rosenberg Self-Esteem Scale; UPPS-P = Impulsive Behavior Scale; VNS = Visual Numerical Scale; MPQ = McGill Pain Questionnaire; PCS = Pain Catastrophizing Scale; MINI = Mini International Neuropsychiatric Interview; OCD = Obsessive Compulsive Disorder; PTSD = Posttraumatic Stress Disorder; GAD: Generalized Anxiety Disorder.

\subsection{Correlation Analysis}

Paranoia scale score positively correlated with BDI-II $(\mathrm{r}=0.738, p<0.001)$, STAI-state $(\mathrm{r}=0.411, p<0.001)$, STAI-trait $(\mathrm{r}=0.691, p<0.001)$, current, average and maximal VNS pain evaluation $(\mathrm{r}=0.409,0.566,0.549$ respectively; $p<$ $0.001)$, sensory and affective MPQ $(\mathrm{r}=0.600,0.658$ respectively; $p<0.001)$, PCS ruminations, magnification and helplessness $(\mathrm{r}=0.554,0.628,0.636$ respectively; $p<0.001)$, pain duration $(\mathrm{r}=0.391, p<0.001)$. Rosenberg self-esteem scale scores negatively correlated with BDI-II $(\mathrm{r}=-0.797, p<0.001)$, STAI-state $(\mathrm{r}=-0.572$, $p<0.001)$, STAI-trait $(\mathrm{r}=-0.852, p<0.001)$, current, average and maximal VNS pain evaluation $(\mathrm{r}=-0.236,-0.381,-0.389$ respectively; $p<0.001)$, sensory and affective MPQ ( $\mathrm{r}=-0.434,-0.563$ respectively; $p<0.001)$, PCS ruminations, magnification and helplessness $(\mathrm{r}=-0.472,-0.487,-0.557$ respectively; $p$ $<0.001)$, pain duration $(\mathrm{r}=-0.319, p<0.01)$. UPPS-P Negative urgency scale score positively correlated with BDI-II $(\mathrm{r}=0.456, p<0.001)$, STAI-state $(\mathrm{r}=$ $0.453, p<0.001)$, STAI-trait $(\mathrm{r}=0.439, p<0.001)$, average and maximal VNS pain evaluation ( $\mathrm{r}=0.289, p=0.01,0.302, p<0.01$ respectively) but not with current VNS ( $\mathrm{r}=0.150, p=0.10)$, sensory and affective MPQ $(\mathrm{r}=0.297,0.289$ respectively; $p<0.01)$, PCS ruminations, magnification and helplessness $(\mathrm{r}=$ $0.328, p<0.01 ; \mathrm{r}=0.420,0.366$ respectively; $p<0.001)$, pain duration $(\mathrm{r}=0.391$, 
$p<0.001)$. UPPS-P Positive urgency scale score positively correlated with BDI-II $(\mathrm{r}=0.249, p=0.025)$, STAI-trait $(\mathrm{r}=0.295, p<0.01)$, sensory and affective MPQ ( $\mathrm{r}=0.251, p=0.022, \mathrm{r}=0.293, p<0.01$ respectively), PCS ruminations, magnification and helplessness $(\mathrm{r}=0.242, p=0.028, \mathrm{r}=0.413, p<0.001 ; \mathrm{r}=$ $0.351, p<0.01$, respectively), pain duration $(\mathrm{r}=0.264, p=0.017)$. UPPS-P Lack of premeditation scale score positively correlated with STAI-trait $(\mathrm{r}=0.266, p=$ $0.016)$ and education level $(\mathrm{r}=0.246, p=0.027)$. UPPS-P Lack of perseverance positively correlated with BDI-II $(\mathrm{r}=0.338, p<0.01)$, STAI-state $(\mathrm{r}=0.411, p<$ $0.001)$, STAI-trait $(\mathrm{r}=0.476, p<0.001)$. Finally, UPPS-P Sensation seeking scale score negatively correlated with STAI-state $(\mathrm{r}=-0.290, p<0.01)$.

The ANCOVA revealed group differences on paranoia as a dependent variable and the following covariates: 1) psychological distress (depression, trait anxiety, state anxiety), $\mathrm{F}=3.654, p=0.031 ; 2$ ) pain severity (intensity of current, mean and maximum pain, pain duration), $\mathrm{F}=13.769, p<0.001$; and 3) education level, $\mathrm{F}=38.890, p<0.001$. An ANCOVA with self-esteem (RSES score) as the dependent variable and psychological distress as a covariate yielded a nonsignificant effect, $\mathrm{F}=1.240, p=0.295$, as did analyses with UPPS-P negative urgency as the dependent variable and psychological distress as the covariate, $\mathrm{F}=0.452, p=$ 0.638 .

\section{Discussion}

Our findings were consistent with the literature data that affective disorders are more prevalent in FM than in other chronic pain conditions (Häuser et al., 2015), as current and past mood and anxiety disorders were more prevalent in the FM group than in the CNP or HC. We found higher depression and state anxiety scores in patients with CNP than in HC, but trait anxiety was only higher in patients with FM. Paranoia scores were significantly higher for FM than for HC, with intermediate scores in CNP, and group differences remained after adjustment for psychological distress (depression and anxiety), pain severity, and education level. Compared with CNP and HC, the FM group had a lower self-esteem score and a higher UPPS-P negative urgency sub score, but this difference could be explained by psychological distress. Moreover, patients with FM described the sensory and affective aspects of perceived pain as being more severe, and expressed higher pain catastrophizing than patients with CNP, suggesting that these aspects should be addressed in nonpharmacological therapies of the illness.

\subsection{Affective Disorder and FM}

Our results suggest that patients with FM are characterized by higher emotion-related distress and more prevalent affective disorders than patients with CNP or HC. We suggest two explanations for this association. First, FM and affective disorders may share the same intermediate phenotype of vulnerability, meaning that FM may be a variant of affective disorder with a predominance of somatic symptoms. Second, affective dysregulation with high depression and 
anxiety may be the consequence of disturbed coping with chronic painful disease.

Based on the biopsychosocial model of FM, it is strongly suggested that psychosocial factors like child abuse or neglect contribute to predisposing, triggering and perpetuating FM symptoms (Häuser et al., 2015). Even though, there is growing number of studies suggesting that FM and depression or somatoform pain disorder are not interchangeable clinical concepts (Häuser \& Fitzcharles, 2018), it is acknowledged that psychosocial stress and emotional conflicts could trigger or aggravate the illness (Häuser \& Henningsen, 2014). The best-established psychopathological features on FM suggest that it is a brain disease with central pain sensitization (Häuser \& Fitzcharles, 2018). We suggest that higher paranoia and lower self-esteem could contribute to poor stress cooping and further central pain sensitization in FM, while the mechanisms of pain maintenance in CNP could be different.

\subsection{Paranoia in Affective Disorders and Chronic Pain}

The main result of our study was a higher level of paranoia in FM compared with the other two groups. Whereas the lower self-esteem in the FM population could be explained by higher levels of anxiety and depression, the higher PS score appeared to be independent of psychological distress, pain severity, and education level as confounding factors.

The literature suggests that in a nonclinical sample, higher trait paranoia and lower self-esteem are significantly correlated with experiential avoidance (Udachina et al., 2009), defined as intolerance toward negatively evaluated bodily sensations, thoughts or emotions, such that individuals make more effort to avoid, suppress or control these mental experiences. Authors further suggest that engaging in these mental control strategies is predictive of paranoid thought occurrence. Experiential avoidance is a theoretical concept that has contributed to the fear-avoidance model of chronic pain, extensively studied in the literature (Kroska, 2016). Moreover, an avoidant behavioral style in the chronic pain population is predictive of higher disability, physical disuse, and depression (Kroska, 2016). Our results suggest that the higher level of paranoia found in FM compared with CNP correlates with the sensory and affective severity of pain, as well as with pain catastrophizing, which could be predictive of functional disability.

High paranoia scores are found in a range of affective disorders, including social phobia (Schutters et al., 2012), posttraumatic stress disorder (Freeman et al., 2012), depression (Wigman et al., 2012), and bipolar disorder (Goodwin \& Jamison, 1990). Moreover, some authors have suggested that specific personality traits such as high harm avoidance and low self-directedness correlate with both the propensity to develop chronic pain (Naylor et al., 2017) and major depressive and bipolar II disorder (Zaninotto et al., 2015).

The high trait paranoia in FM may therefore reflect activated schemas of a negative self and hostile others, combined with difficulty reappraising situations 
in order to effectively downregulate trait anxiety and other negative emotions in the face of pain. Coping with disease appeared less maladaptive in patients with CNP, in terms of emotion-related processes. We suggest that in FM, chronic pain perception is related to central sensitization induced by a preexisting affective disorder, whereas in CNP, emotion-related distress is the consequence of the cognitive integration of chronic pain. Moreover, a high prevalence of FM-like symptoms (musculoskeletal pain, disturbed sleep and fatigue) is associated with paranoid ideation in veterans with posttraumatic stress syndrome (Moldofsky et al., 2016), suggesting that an affective disorder can be the cause of somatic complaints. Therefore, the hypothesis that FM is a primary affective disorder should be addressed in future studies, in order to identify endophenotype markers and explore the time course of emotion-related distress.

\subsection{Limitations and Conclusion}

Our study had several limitations. First, this is a cross-sectional comparison of the relatively small samples that limits further generalization of our results. Second, responses to self-report questionnaires can be biased by participants' personal feelings and their motivation for taking part in the study. Third, our sample was consecutively recruited in naturalistic conditions, in hospital units of pain management, and not perfectly matched. These aspects could limit further generalization and reliability of our findings. In addition, our findings could have other interpretations, like differential social acceptance and FM-associated stigma that could contribute to higher paranoia. Nevertheless, this is the first study that reports higher paranoia scores in FM patients. While prospective studies have demonstrated different trajectories of the relationship chronic pain-depression (Zhu et al., 2014), it would be interesting to explore if higher paranoia trait is consistent in otherwise heterogeneous profile of FM patients.

In conclusion, this preliminary study revealed significantly higher emotion-related distress in FM compared with CNP and HC. We suggest that FM and CNP do not share the same intermediate phenotype. Future studies should undertake comparisons between patients with mood disorders, particularly major depression and bipolar II disorder, and patients with FM on the paranoia, self-esteem and impulsivity trait dimensions. Findings from this pilot study may help improve the psychological evaluation associated to diagnostic and therapeutic challenges of chronic pain management in general and in FM management that requires multidisciplinary approaches in particular.

\section{Acknowledgements}

We would like to thank all participants to this study.

\section{Conflicts of Interest}

The authors declare no conflicts of interest regarding the publication of this paper. 


\section{References}

Baker, R. (2007). Emotional Processing: Healing through Feeling. Oxford: Lion-Hudson.

Baker, R., D’Mello, S., Rodrigo, M., \& Graesser, A. (2010). Better to Be Frustrated than Bored: The Incidence and Persistence of Affect during Interactions with Three Different Computer-Based Learning Environments. International Journal of Human-Computer Studies, 68, 223-241. https://doi.org/10.1016/j.ijhcs.2009.12.003

Bar-On Kalfon, T., Gal, G., Shorer, R., \& Ablin, J. N. (2016). Cognitive Functioning in Fibromyalgia: The Central Role of Effort. Journal of Psychosomatic Research, 87, 30-36. https://doi.org/10.1016/j.jpsychores.2016.06.004

Beck, A. T., Steer, R. A., \& Brown, G. K. (1996). Manual for Beck Depression Inventory II (BDI-II). San Antonio, TX: Psychology Corporation. https://doi.org/10.1037/t00742-000

Bentall, R. P., Rowse, G., Shryane, N., Kinderman, P., Howard, R., Blackwood, N., Moore, R., \& Corcoran, R. (2009). The Cognitive and Affective Structure of Paranoid Delusions: A Transdiagnostic Investigation of Patients with Schizophrenia Spectrum Disorders and Depression. Archives of General Psychiatry, 66, 236-247. https://doi.org/10.1001/archgenpsychiatry.2009.1

Billieux, J., Rochat, L., Ceschi, G., Carré, A., Offerlin-Meyer, I., Defeldre, A. C., Khazaal, Y., Besche-Richard, C., \& Van der Linden, M. (2012). Validation of a Short French Version of the UPPS-P Impulsive Behavior Scale. Comprehensive Psychiatry, 53, 609-615. https://doi.org/10.1016/j.comppsych.2011.09.001

Conrad, R., Schilling, G., Bausch, C., Nadstawek, J., Wartenberg, H. C., Wegener, I., Geiser, F., Imbierowicz, K., \& Liedtke, R. (2007). Temperament and Character Personality Profiles and Personality Disorders in Chronic Pain Patients. Pain, 15, 197-209. https://doi.org/10.1016/j.pain.2007.07.024

Fenigstein, A., \& Vanable, P. A. (1992). Paranoia and Self-Consciousness. Journal of Personality and Social Psychology, 62, 129-138.

https://doi.org/10.1037/0022-3514.62.1.129

Freeman, D., Stahl, D., McManus, S., Meltzer, H., Brugha, T., Wiles, N., \& Bebbington, P. (2012). Insomnia, Worry, Anxiety and Depression as Predictors of the Occurrence and Persistence of Paranoid Thinking. Social Psychiatry and Psychiatric Epidemiology, 47, 1195-1203. https://doi.org/10.1007/s00127-011-0433-1

Galvez-Sánchez, C. M., Reyes Del Paso, G. A., \& Duschek, S. (2018). Cognitive Impairments in Fibromyalgia Syndrome: Associations with Positive and Negative Affect, Alexithymia, Pain Catastrophizing and Self-Esteem. Frontiers in Psychology, 9, 377. https://doi.org/10.3389/fpsyg.2018.00377

Goodwin, F., \& Jamison, K. (1990). Manic-Depressive Illness. Oxford: Oxford University Press.

Häuser, W., \& Fitzcharles, M. A. (2018). Facts and Myths Pertaining to Fibromyalgia. Dialogues in Clinical Neuroscience, 20, 53-62. https://doi.org/10.31887/DCNS.2018.20.1/whauser

Häuser, W., \& Henningsen, P. (2014). Fibromyalgia Syndrome: A Somatoform Disorder? European Journal of Pain, 18, 1052-1059. https://doi.org/10.1002/j.1532-2149.2014.00453.x

Häuser, W., Ablin, J., Fitzcharles, M. A., Littlejohn, G., Luciano, J. V., Usui, C., \& Walitt, B. (2015). Fibromyalgia. Nature Reviews Disease Primers, 13, Article No. 15022. https://doi.org/10.1038/nrdp.2015.22

Hopwood, C. J., Wright, A. G., Krueger, R. F., Schade, N., Markon, K. E., \& Morey, L. C. 
(2013). DSM-5 Pathological Personality Traits and the Personality Assessment Inventory. Assessment, 20, 269-285. https://doi.org/10.1177/1073191113486286

Kroska, E. B. (2016). A Meta-Analysis of Fear-Avoidance and Pain Intensity: The Paradox of Chronic Pain. Scandinavian Journal of Pain, 13, 43-58. https://doi.org/10.1016/j.sjpain.2016.06.011

Lumley, M. A., Cohen, J. L., Borszcz, G. S., Cano, A., Radcliffe, A. M., Porter, L. S. et al (2011). Pain and Emotion: A Biopsychosocial Review of Recent Research. Journal of Clinical Psychology, 67, 942-968. https://doi.org/10.1002/jclp.20816

Mansour, A. R., Farmer, M. A., Baliki, M. N., \& Apkarian, A. V. (2014). Chronic Pain: The Role of Learning and Brain Plasticity. Restorative Neurology and Neuroscience, 32, 129-139.

Melzack, R. (1975). The McGill Pain Questionnaire: Major Properties and Scoring Methods. Pain, 1, 277-299. https://doi.org/10.1016/0304-3959(75)90044-5

Moldofsky, H., Rothman, L., Kleinman, R., Rhind, S. G., \& Richardson, J. D. (2016). Disturbed EEG Sleep, Paranoid Cognition and Somatic Symptoms Identify Veterans with Post-Traumatic Stress Disorder. BJPsych Open, 2, 359-365.

https://doi.org/10.1192/bjpo.bp.116.003483

Naylor, B., Boag, S., \& Gustin, S. M. (2017). New Evidence for a Pain Personality? A Critical Review of the Last 120 Years of Pain and Personality. Scandinavian Journal of Pain, 17, 58-67. https://doi.org/10.1016/j.sjpain.2017.07.011

Prokofieva, V., Kostromina, S., Polevaia, S., \& Fenouillet, F. (2019). Understanding Emotion-Related Processes in Classroom Activities through Functional Measurements. Frontiers in Psychology, 10, 2263. https://doi.org/10.3389/fpsyg.2019.02263

Rosenberg, M. (1965). Society and the Adolescent Self-Image. Princeton, NJ: Princeton University Press. https://doi.org/10.1515/9781400876136

Rosselló, F., Muñoz, M. A., Duschek, S., \& Montoya, P. (2015). Affective Modulation of Brain and Autonomic Responses in Patients with Fibromyalgia. Psychosomatic Medicine, 77, 721-732. https://doi.org/10.1097/PSY.0000000000000217

Schutters, S. I. J., Dominguez, M. D. G., Knappe, S., Lieb, R., van Os, J., Schruers, K. R. J., \& Wittchen, H. U. (2012). The Association between Social Phobia, Social Anxiety Cognitions and Paranoid Symptoms. Acta Psychiatrica Scandinavica, 125, 213-227. https://doi.org/10.1111/j.1600-0447.2011.01787.x

Sheehan, D. V., Lecrubier, Y., Sheehan, K. H., Amorim, P., Janavs, J., Weiller, E., Hergueta, T., Baker, R., \& Dunbar, G. C. (1998). The Mini-International Neuropsychiatric Interview (M.I.N.I.): The Development and Validation of a Structured Diagnostic Psychiatric Interview for DSM-IV and ICD-10. Journal of Clinical Psychiatry, 59, 22-33.

Spielberger, C. D., Gorsuch, R. L., \& Lushene, R. E. (1984). STAI Manual for the State-Trait Anxiety Inventory: Self-Evaluation Questionnaire. Palo Alto, CA: Consulting Psychologists Press.

Sullivan, M. J. L., Bishop, S., \& Pivik, J. (1995). The Pain Catastrophizing Scale: Development and Validation. Psychological Assessment, 7, 432-524. https://doi.org/10.1037/1040-3590.7.4.524

Thewissen, V., Bentall, R. P., Oorschot, M., A Campo, J., van Lierop, T., van Os, J., \& Myin-Germeys, I. (2011). Emotions, Self-Esteem, and Paranoid Episodes: An Experience Sampling Study. British Journal of Clinical Psychology, 50, 178-195.

Udachina, A., Thewissen, V., Myin-Germeys, I., Fitzpatrick, S., O’Kane, A., \& Bentall, R. P. (2009). Understanding the Relationships between Self-Esteem, Experiential Avoidance, and Paranoia: Structural Equation Modelling and Experience Sampling Studies. 
The Journal of Nervous and Mental Disease, 197, 661-668. https://doi.org/10.1097/NMD.0b013e3181b3b2ef

Wigman, J. T., van Nierop, M., Vollebergh, W. A., Lieb, R., Beesdo-Baum, K., Wittchen, H. U., \& Van Os, J. (2012). Evidence That Psychotic Symptoms Are Prevalent in Disorders of Anxiety and Depression, Impacting on Illness Onset, Risk, and Severity-Implications for Diagnosis and Ultra-High Risk Research. Schizophrenia Bulletin, 38, 247-257. https://doi.org/10.1093/schbul/sbr196

Woolf, B., Burleson, W., Arroyo, I., Dragon, T., Cooper, D., \& Picard, R. (2009). Affect-Aware Tutors: Recognising and Responding to Student Affect. International Journal of Learning Technology, 4, 129-164. https://doi.org/10.1504/IJLT.2009.028804

Yılmaz, E., \& Tamam, L. (2018). Attention-Deficit Hyperactivity Disorder and Impulsivity in Female Patients with Fibromyalgia. Neuropsychiatric Disease and Treatment, 14, 1883-1889. https://doi.org/10.2147/NDT.S159312

Zaninotto, L., Souery, D., Calati, R., Di Nicola, M., Montgomery, S., Kasper, S., Zohar, J., Mendlewicz, J., Robert Cloninger, C., Serretti, A., \& Janiri, L. (2015). Temperament and Character Profiles in Bipolar I, Bipolar II and Major Depressive Disorder: Impact over Illness Course, Comorbidity Pattern and Psychopathological Features of Depression. Journal of Affective Disorders, 184, 51-59.

https://doi.org/10.1016/j.jad.2015.05.036

Zhu, Z., Galatzer-Levy, I. R., \& Bonanno, G. A. (2014). Heterogeneous Depression Responses to chronic Pain Onset among Middle-Aged Adults: A Prospective Study. Psychiatry Research, 217, 60-66. https://doi.org/10.1016/j.psychres.2014.03.004

Zisner, A., \& Beauchaine, T. P. (2016). Neural Substrates of Trait Impulsivity, Anhedonia, and Irritability: Mechanisms of Heterotypic Comorbidity between Externalizing Disorders and Unipolar Depression. Development and Psychopathology, 28, 1177-1208. https://doi.org/10.1017/S0954579416000754 